\title{
Improving the Performance of a Cycloidal Coded-Aperture Miniature Mass Spectrometer
}

\section{Supplementary Material}

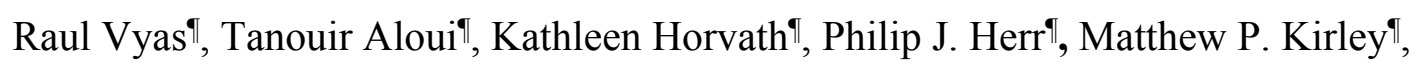
Charles B. Parker", Adam D. Keil\&, James B. Carlson $\$$, Justin Keogh*, Roger P. Sperline*, M. Bonner Denton*, M. Luisa Sartorelli^|a, Brian R. Stonerף, Michael E. Gehmף, Jeffrey T. Glass", Jason J. Amsdenף

\section{Author Affiliations:}

ฯ Department of Electrical and Computer Engineering, Duke University, Durham, NC, USA 27708

\& Broadway Analytical, LLC, Monmouth, IL

$\$$ Engineering and Applied Physics Division, RTI International, Research Triangle Park, NC USA 27709

*Department of Chemistry and Biochemistry, University of Arizona, Tucson, AZ USA 85721

aDepartamento de Física, Universidade Federal de Santa Catarina, Campus Universitário Trindade, 88040-000 Florianópolis, SC BRAZIL

\section{${ }^{\wedge}$ Corresponding Author:}

Jason J. Amsden 
Department of Electrical and Computer Engineering

Duke University

Durham, NC 27708

(919)660-5592

jason.amsden@duke.edu 


\section{Supplemental Material}

This material covers some details from the new C-CAMMS-MP system not expanded upon in the main text of the paper. In this document, the main emphasis is on the differences in the ion source and mass analyzer designs between the new C-CAMMS-MP and the previously reported proof-of-concept C-CAMMS instrument [1].

\subsection{C-CAMMS-MP ion source and detector assembly}

Figure S1 presents 3-D CAD models of the new C-CAMMS-MP and the previous CCAMMS mass analyzers. Assembly of the ion source and detector within the electric sector electrodes of the C-CAMMS-MP mass analyzer is a four-step process. First, the ion repeller is adhered to a printed circuit board (PCB) via conductive silver epoxy (EPOTEK EE129-4). Next, the ion repeller assembly is then placed in a slot within the bottom electrode (Electrode 12) and attached to it via non-conductive epoxy (Loctite EA E-60 epoxy). Then, the coded aperture is adhered to the top electrode (Electrode 13) via conductive silver epoxy. Finally, the detector is then placed at the mass analyzer focal plane within a cavity in Electrode 13. Since the coded aperture and the detector planes are aligned with the mass analyzer focal plane, this improves the depth-of-focus of the new C-CAMMS-MP. Figure S2 compares the ion source and the detector assembled within the electric sectors of the previous C-CAMMS and the new C-CAMMS-MP instruments. 


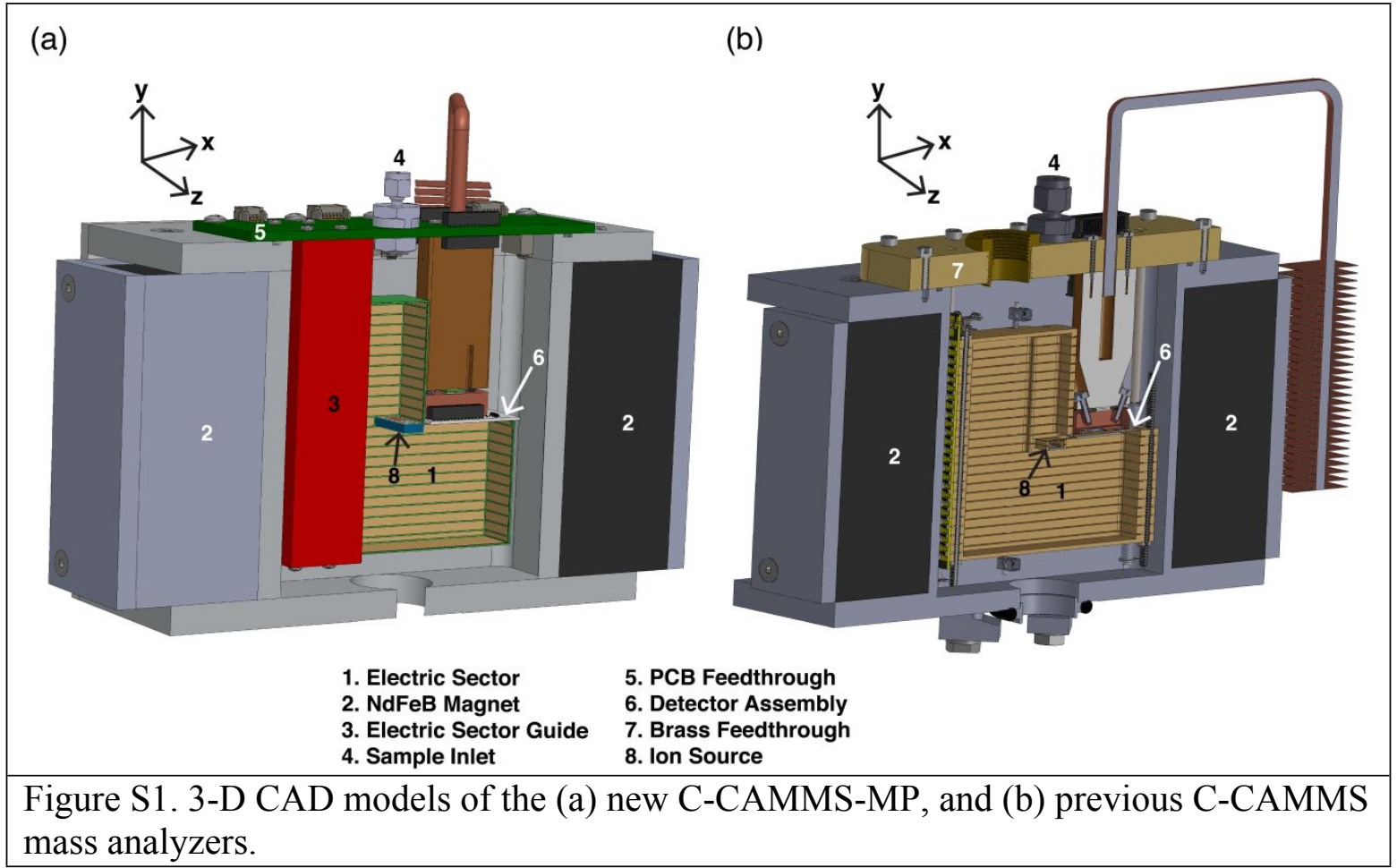

Figure $\mathrm{S} 3$ presents cross-sections of the $\mathrm{CAD}$ of the previous C-CAMMS and the new $\mathrm{C}$ CAMMS-MP electric sectors, comparing the positioning of the ion source and the detector in the two instruments relative to their electric sector electrodes. Note that the green circuit board solder mask in the previous C-CAMMS CAD image (Figure S2(b)) appears to be present in between the electrode gold tracks in the corners, which would make the electric sector electrodes discontinuous. However, in the hardware implementation of the previous C-CAMMS electric sector, the gold tracks were soldered (at each of the perpendicular joints where the circuit boards meet) to ensure electrical continuity for each electrode. 


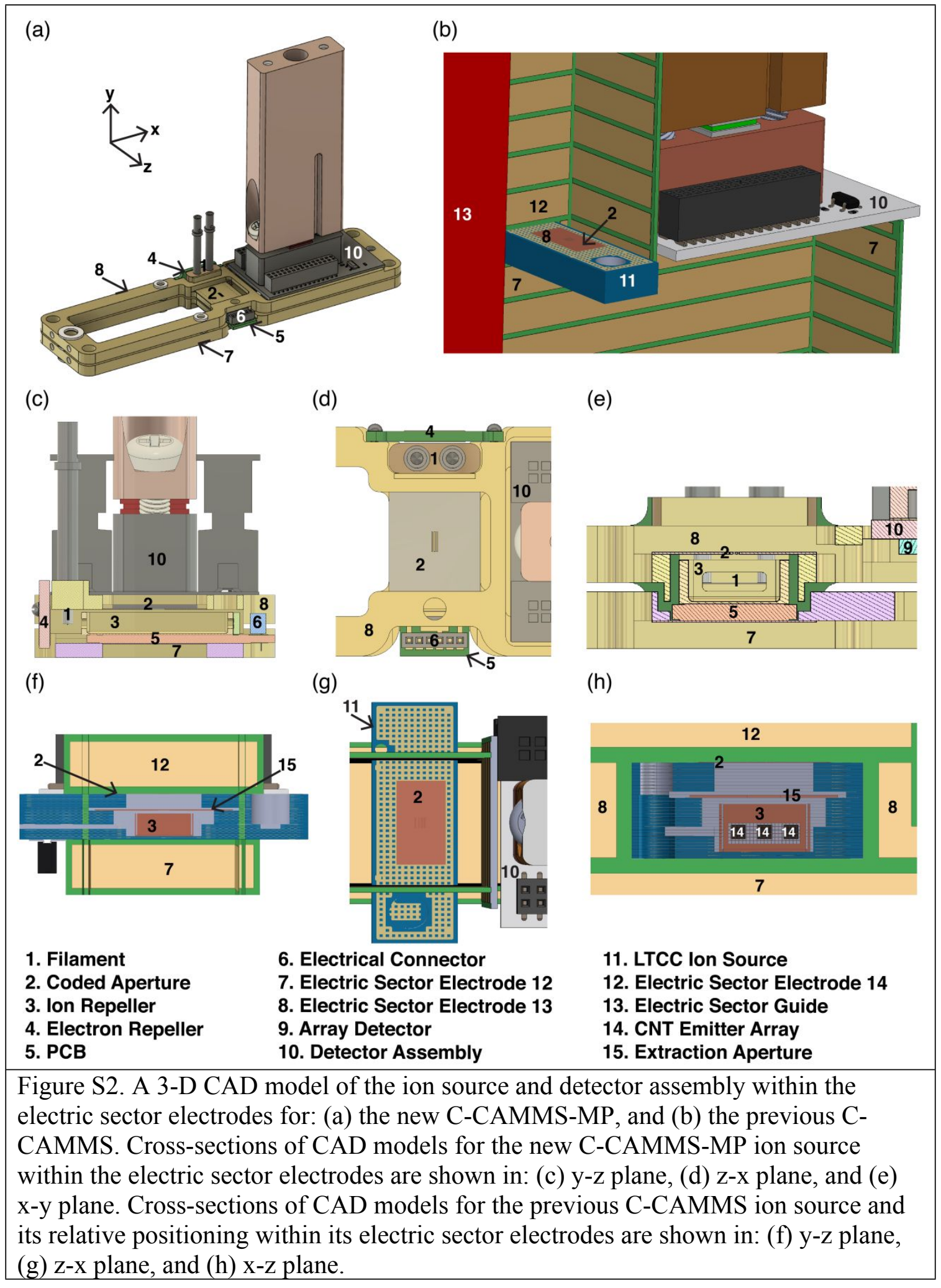


(a)

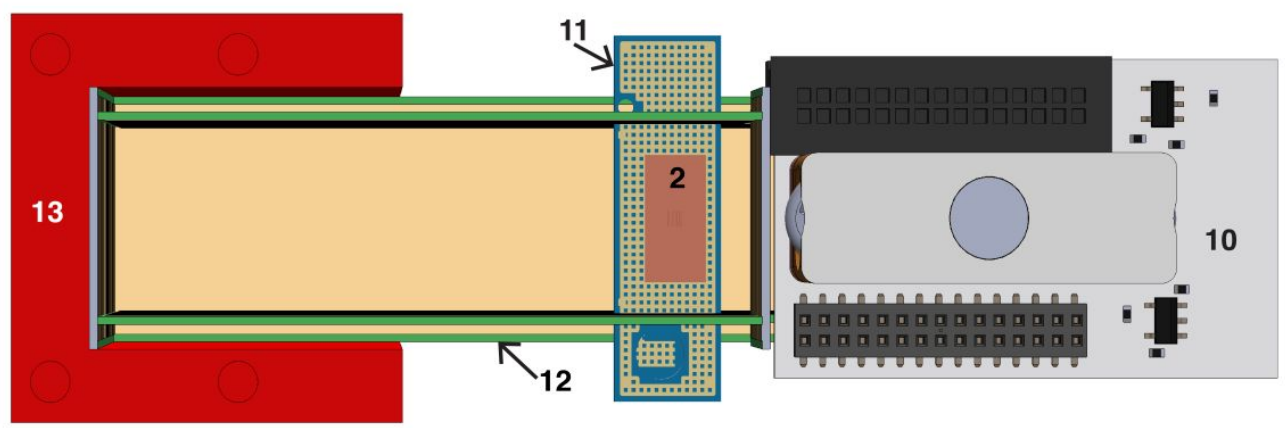

(b)

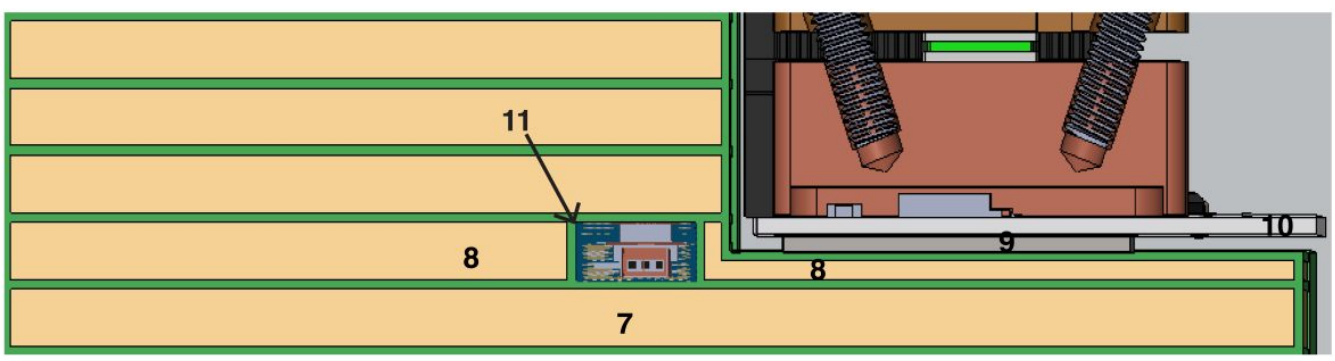

(c)

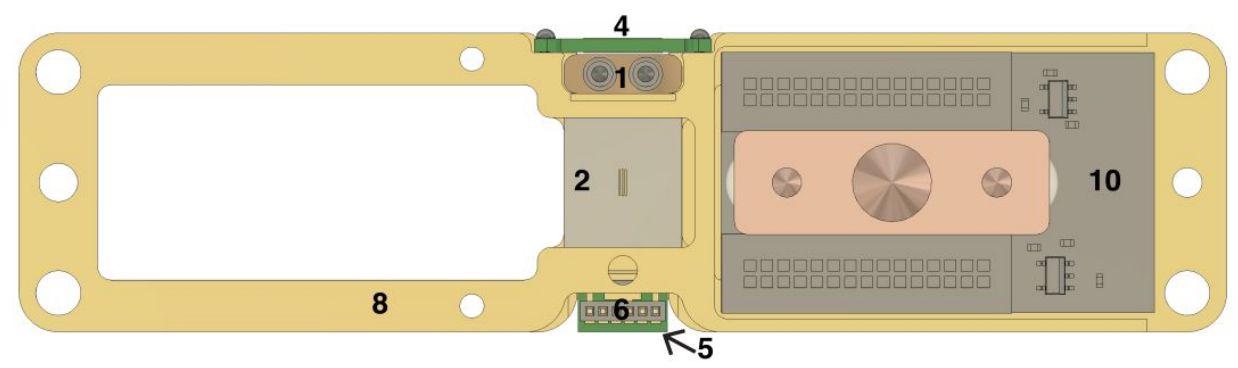

(d)

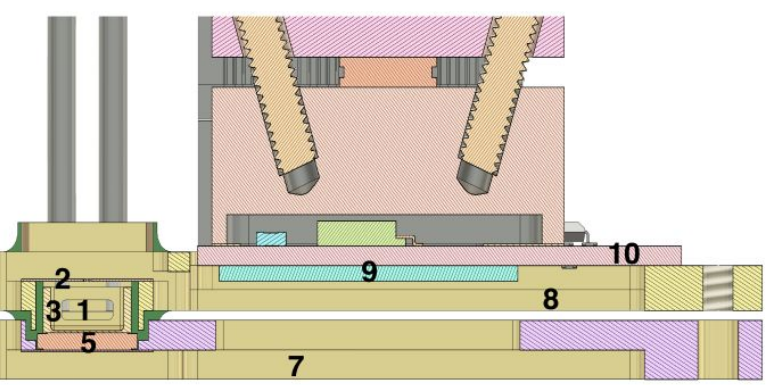

1. Filament

2. Coded Aperture

3. Ion Repeller

4. Electron Repeller

5. РСB
8

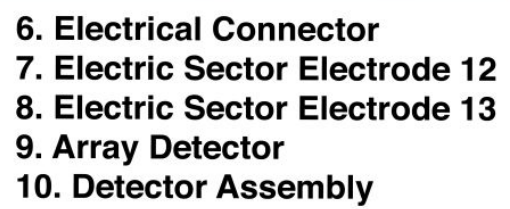

11. LTCC Ion Source

12. Electric Sector

13. Electric Sector Guide

14. CNT Emitter Array

Figure S3. Cross-section of the CAD models for the previous C-CAMMS ion source and the detector and their relative positioning within its electric sector electrodes are shown in: (a) z-x plane, and (b) x-y plane. Cross-sections of CAD models for the new C-CAMMS-MP ion source and the detector assembly within the electric sector electrodes are shown in: (c) z-x plane, and (d) x-y plane. 


\subsection{Proof-of-concept C-CAMMS vs. C-CAMMS-MP ion source simulations}

Figure S4 shows electron and ion trajectories corresponding to (a) previous C-CAMMS ion source, with an average ion energy of $26.5 \pm 0.9 \mathrm{eV}$, and (b) C-CAMMS-MP ion source, with an average ion energy of $27.4 \pm 0.4 \mathrm{eV}$ indicating a lower energy dispersion. The new C-CAMMS-MP ion source also produced lower angular dispersion of the ions exiting the aperture plane, over that of the previous C-CAMMS ion source. The colorcoded ion trajectories represent simulated ion energies. Removal of the extraction aperture in the new C-CAMMS-MP ion source indicated its minimal impact on ion energies, expressed in an average ion energy similar to the previous C-CAMMS ion source ( $26.5 \mathrm{eV}$ vs. $27.4 \mathrm{eV}$, respectively).

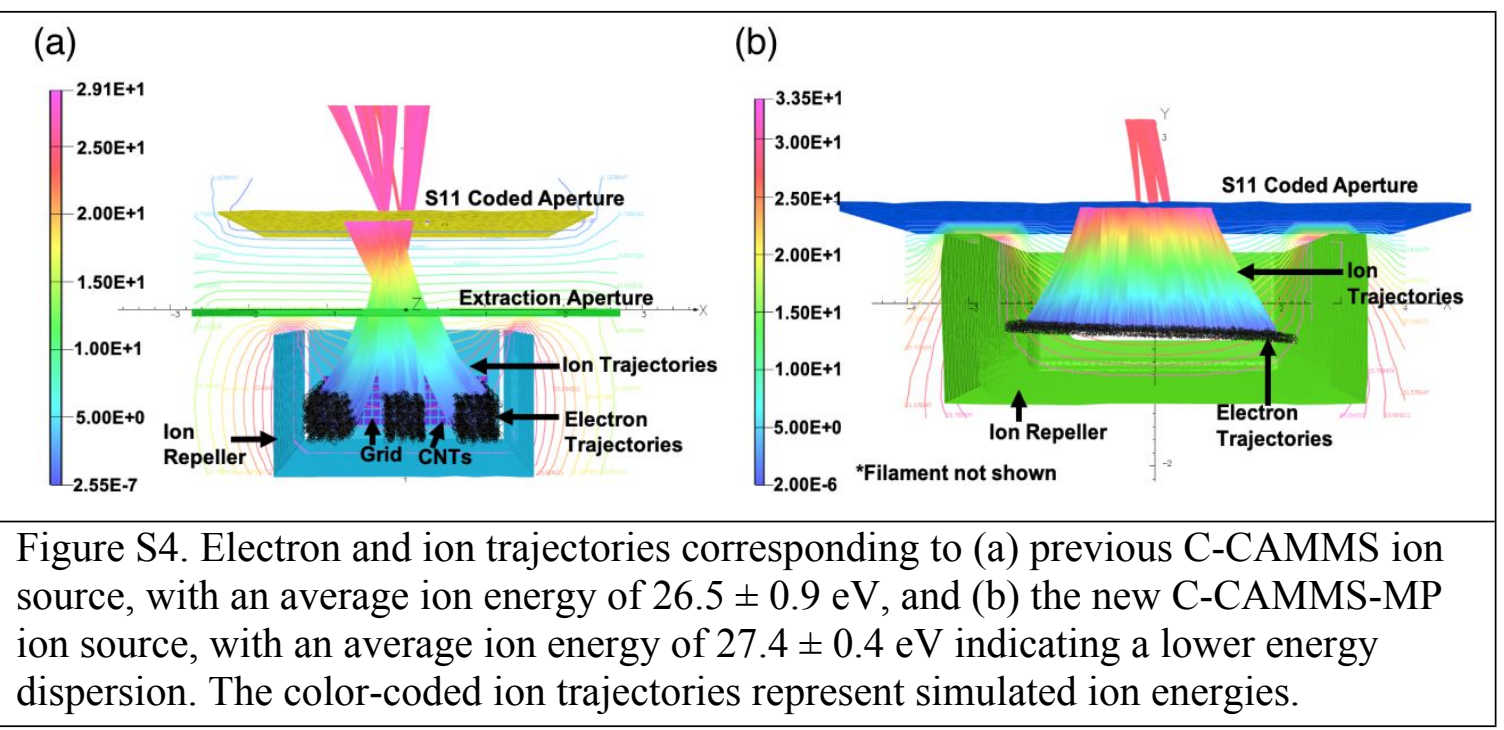




\subsection{Mass Analyzer CAD Images}

(a)

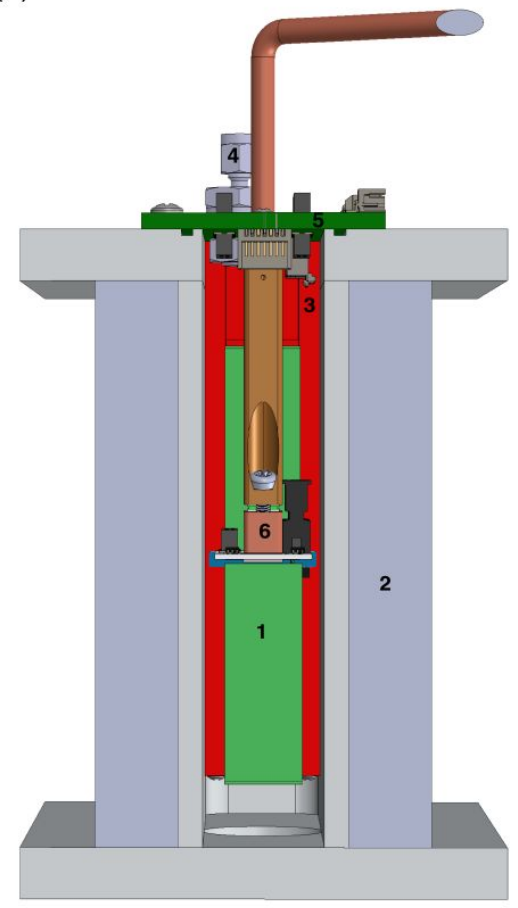

(b)

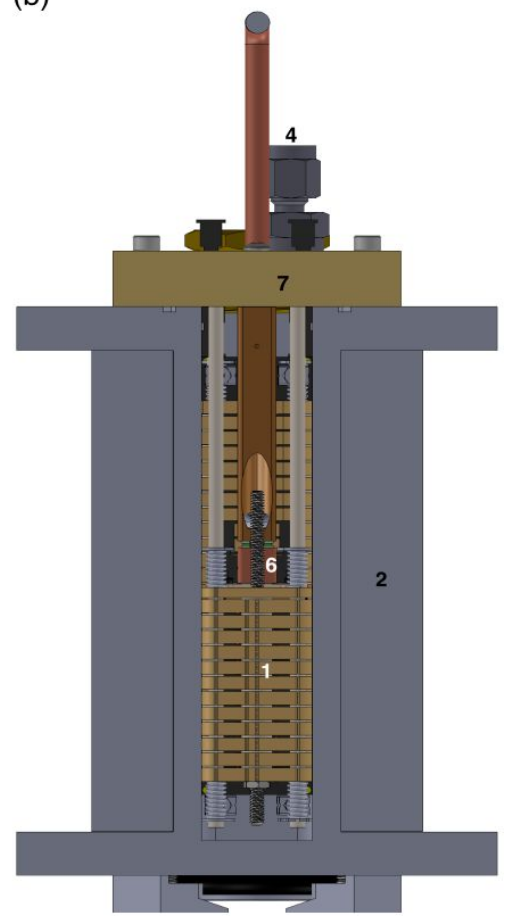

(c)
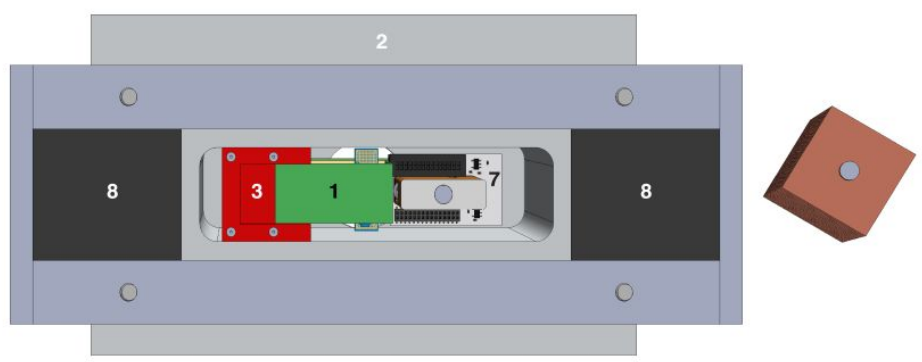

(d)

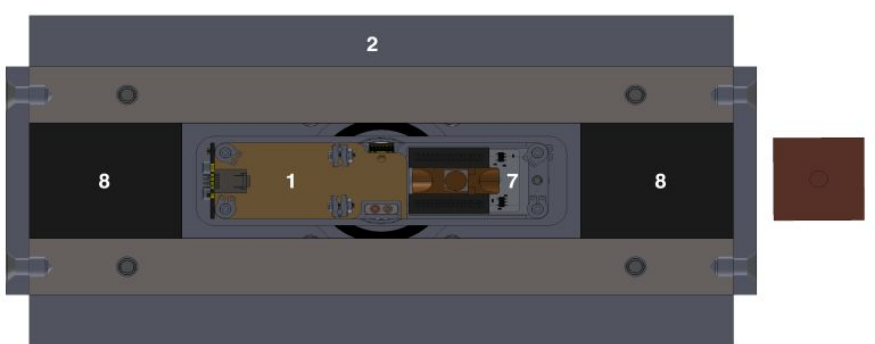

1. Electric Sector

2. Vacuum Manifold

3. Electric Sector Guide

5. PCB Feedthrough 6. Detector Assembly

4. Sample Inlet

7. Brass Feedthrough

Figure S5. Cross-section of the CAD model for the previous CCAMMS mass analyzer in (a) y-z plane, and (c) z-x plane. Crosssection of the CAD model for the new C-CAMMS-MP mass analyzer in (a) y-z plane, and (c) z-x plane. 


\subsection{References}

1. Amsden, J.J., et al., Proof of concept coded aperture miniature mass spectrometer using a cycloidal sector mass analyzer, a carbon nanotube (CNT) field emission electron ionization source, and an array detector. Journal of The American Society for Mass Spectrometry, 2018. 29(2): p. 360-372. 